BMJ Open

Sport \&

Exercise

Medicine

\title{
Physical activity levels, lifestyle behaviour and musculoskeletal health profiles among seated video gamers during COVID-19
}

\author{
Nicholas Harding, Habib Noorbhai
}

\begin{abstract}
To cite: Harding N, Noorbhai $\mathrm{H}$. Physical activity levels, lifestyle behaviour and musculoskeletal health profiles among seated video gamers during COVID-19. BMJ Open Sport \& Exercise Medicine 2021;7:e001194. doi:10.1136/ bmjsem-2021-001194
\end{abstract}

Accepted 23 August 2021

\section{Check for updates}

(c) Author(s) (or their employer(s)) 2021. Re-use permitted under CC BY-NC. No commercial re-use. See rights and permissions. Published by BMJ.

Biomedical Engineering and Healthcare Technology (BEAHT) Research Centre, Faculty of Health Sciences, University of Johannesburg, Johannesburg, Gauteng, South Africa

Correspondence to Professor Habib Noorbhai; habibn@uj.ac.za

\section{ABSTRACT}

Objectives The aim of this research study was to determine the physical activity levels of non-active video gamers, and to determine how much other time was spent with sedentary behaviours, both in recreational and occupational domains.

Methods The study used the International Physical Activity Questionnaire, and investigated physical activity levels and health data of seated gamers. Typical weekly gaming habits, self-reported musculoskeletal pain and physical activity levels experienced by participants while gaming was also obtained.

Results Out of a total of 102 participants, $69.6 \%$ stated that they game for 3 hours or more on a typical weekday. Most participants video game seated or reclined $(96.1 \%$, $p=0.01$ ), with only four participants engaging in some form of active video gaming. In relation to physical activity levels, despite the high frequency and duration of gameplay, $87.3 \%$ of participants stated they engaged in moderate-intensity physical activity $(p=0.02)$. There was an association with video gaming and musculoskeletal pain, with over half of the participants stating they experienced pain while gaming. There was also a link between seated video gaming and musculoskeletal pain among participants.

Conclusion Findings from this study show that the high frequency and duration of video gaming among gamers does not affect physical activity for moderate and vigorous intensities, but does affect musculoskeletal pain. Further research is required to determine whether video gaming has a significant effect on lifestyle, sedentary habits and musculoskeletal health, especially in the context of the COVID-19 pandemic where sedentary behaviour has likely increased.

\section{INTRODUCTION}

Video games are electronically based forms of entertainment, played on television, computers and other electronic platforms, with the key element being human interaction with the electronic platform invoking visual feedback. It is a prevalent pastime activity, today, with a growth rate of $10.7 \%$ in the year 2018. ${ }^{1}$ In the USA alone, it was found that 162 million people own video

\section{Key messages}

What is already known?

- There is a link between sedentary behaviour and poor musculoskeletal health; specifically, prolonged sedentary behaviour and physical inactivity.

- Previous studies regarding video gameplay, and its contribution to sedentary behaviour have solely focused on adolescents and the youth, but have not included adults, who make up the greater proportion of modern-day video gamers.

\section{What are the new findings?}

- There is a link between seated video gaming and musculoskeletal pain among participants. High frequency and duration for video gaming among gamers does not affect physical activity for moderate and vigorous intensities, but does affect musculoskeletal pain.

- Being conscious of good posture is rarely prioritised by seated video gamers. A vast majority stated that they did not make use of any aids to assist them with good posture.

gaming consoles; $70 \%$ of those being adults. ${ }^{2}$ Worldwide, almost 3 billion people engage in video gaming, with China, France, Germany, Japan and the USA making up the countries with the highest concentration of gamers and most revenue spent. ${ }^{3}$ Competitive gaming, known as eSports, is a billion dollar enterprise with a growth rate of $14 \%$ each year and has an estimated fan base of 454 million fans across the world. ${ }^{4}$ A study conducted across several countries including the USA and UK, found that the mobile phone was the most popular device to game on, followed by the computer (laptop or desktop) and gaming consoles (Xbox, Playstation, etc), with the least popular being the Tablet. ${ }^{5}$

Non-active video gaming and active video gaming are two different forms of gamification. Active video games can be defined as 'video games that require physical activity to play' and non-active video games are defined 
as 'video games that are played sedentary'. ${ }^{6}$ In addition, non-active gaming, a sedentary behaviour (any waking behaviour characterised by an energy expenditure $\leq 1.5$ metabolic equivalents, while in a sitting, reclining or lying posture) as compared with active video gaming, has negative health implications including weight gain, and an increase in the amount of snacking of sugary foods. ${ }^{6}{ }^{7}$ Furthermore, the study associates active video gaming with a higher total energy expenditure. Nonactive video gaming as a sedentary behaviour increases the risk of developing metabolic syndrome, a disease defined as 'central obesity (waist circumference) plus any two of the following four risk factors: raised blood pressure (systolic $\geq 130$ or diastolic $\geq 85$ ), raised triglycerides $(\geq 150 \mathrm{mg} / \mathrm{dL})$, reduced high density lipoprotein (HDL) cholesterol $(<40 \mathrm{mg} / \mathrm{dL}$ in males and $<50 \mathrm{mg} / \mathrm{dL}$ in females) and raised fasting plasma glucose $(\geq 100 \mathrm{mg} /$ $\mathrm{dL}){ }^{8}$. Metabolic syndrome and the associated risk factors increase the risk of myocardial infarction, coronary artery disease and type 2 diabetes. ${ }^{9}$ It is desirable to be more physically active than sedentary, with physical activity diminishing the risks of chronic diseases, such as non-insulin resistant diabetes and early-onset disability or disability later in life. ${ }^{10}$

\section{Weight and obesity}

Physical activity and exercise promote energy expenditure (burning of calories), and helps control an individual's appetite by preventing overeating, making it a well-documented means of maintaining or losing weight. ${ }^{11}$ In modern society, physical activity is no longer as prevalent, given the increase in sedentary behaviour (ie, such as seated video gaming), leading to higher rates of overweight and obesity. ${ }^{12}$ These behaviours are of concern and on the rise, especially due to the COVID-19 pandemic, physical distancing measures and varied lockdown levels in each country. Overweight and, more specifically, obesity is a limiting factor in being able to perform physical activities. This is a major challenge only exacerbated by increased sedentary habits such as seated video gaming. ${ }^{13}$

\section{Sedentary behaviour}

It is a well-established fact that sedentary behaviour is increasing in prevalence. In previous eras, physical activity was more of a necessity than it is currently. ${ }^{11} 12$ Previously, people walked longer distances to access public transport, and physical labour was more demanding as they did not have the technological advancements that are present in modern society. More specifically the development of smartphones, televisions and video gaming consoles have resulted in reduced active recreational activities and hobbies, replacing them with more sedentary or seated hobbies, thereby contributing to the increase in sedentary behaviour, physical inactivity and non-communicable diseases (NCDs) in modern society. Cancer is an NCD (not contagious) that has the second highest mortality rate worldwide, second to heart disease. ${ }^{14}$ Growing evidence suggests a link between the formation of various cancers, and sedentary behaviour. These cancers include colorectal ovarian, endometrial and lung cancer. Seated activities such as non-active video gaming to be statistically associated with the prevalence of colon and rectal cancers. The importance of exercise, specifically aerobic training in preventing colon and rectal cancers is further highlighted. ${ }^{15}$

\section{Measuring sedentary behaviour}

The relationship between physical activity ('any bodily movement produced by skeletal muscles that requires energy expenditure ${ }^{14}$ ) and sedentary behaviour per week is of paramount importance. The American College of Sports Medicine (ACSM) recommends that adults should engage in at least $30 \mathrm{~min}$ of moderate intensity activity 5 days/week $(150 \mathrm{~min})$ or $20 \mathrm{~min}$ of vigorous intensity activity, three times per week $(60 \mathrm{~min})$ in order to help maintain adequate health. Included, is recommendations for strength or resistance training, which should be done two times per week. ${ }^{16}$ NCDs are found to be a significant cause of death in developed and lowincome and middle-income countries. ${ }^{17}$ These NCDs can include cardiovascular diseases, cancers, diabetes and lung diseases all of which occur from poor lifestyle choices including unhealthy eating, inadequate exercise and smoking coupled with increased sedentary activities. Increasing physical activity levels to the standards of the ACSM guidelines can lead to a reduction in deaths caused by NCDs.

In order to measure the relationship between sedentary behaviour and physical activity, one can make use of the International Physical Activity Questionnaire (I-PAQ), which has been approved and used in 12 different countries to document and compare physical activity levels and behaviours. More specific to our study, one can measure the sedentary activity of non-active video gaming with the use of questions from the I-PAQ. The I-PAQ measures the amount of moderate and vigorous activity an individual engages in on a weekly basis. ${ }^{18}$ The I-PAQ is also practical (especially in situations where extensive research is required), low cost and easy to administer. ${ }^{10}$

\section{Association of sedentary behaviour with mental health}

Increased sedentary behaviour and reduced physical activity levels are associated with substandard mental health. However, it is unknown if all sedentary activities have this association. Furthermore, a study highlighted an association between low physical activity levels and increased rates of depression (based on a trial over a 2-week period), and that an increase of sedentary behaviour by $32 \mathrm{~min} /$ day impacted mental health and overall mood. ${ }^{19}$ Regarding treatment, for serious mental health disorders, such as major depression, schizophrenia and bipolar disorder, interventions involving reducing sedentary behaviour and increasing physical activity levels have been deemed to be effective. A study conducted in Australia involving the use of accredited exercise 
physiologists implementing exercise programmes for patients suffering mental disorders, found that exercise programmes and sedentary behaviour reduction interventions to be effective treatment modalities of mental disorders, further finding methodical physical activity to reduce feelings of depression and anxiety, as well as improving sleep patterns. ${ }^{20}$

\section{Musculoskeletal health}

Sedentary behaviour is also associated with musculoskeletal pain. ${ }^{21}$ Additionally, there is also a link with sedentary behaviour and poor musculoskeletal health. Specifically, prolonged sedentary behaviour and physical inactivity, which relates to muscle strength training and this increases the risk of developing sarcopenia (the loss of overall musculature and strength), osteoporosis (decrease in bone density) and osteoarthritis (joint degeneration). ${ }^{22}$ Modern society and the nature of the corporate world has led to lengthened amounts of time being spent seated at a desk working. This is coupled with an increase in sedentary hobbies such as seated video gaming. More time is spent being sedentary, causing weight gain and the development of certain NCDs. The increase in weight gain causes an increase in joint and muscle strain on the body, therefore increasing the prevalence of lower back pain. Lower back pain can be prevented or managed through exercise; however, the reality is that most people lead stressful lives that demotivate them to exercise. Not to mention the lack of time being a contributing factor of people not partaking in exercise. ${ }^{23}$ These pose challenges in modern society. Finding the time for regular exercise can greatly diminish the risks of developing poor musculoskeletal health and help alleviate pain and discomfort related to prolonged seated activities. A 2019 review of current physical activity recommendations shows that exercise can diminish musculoskeletal pains in the shoulders, neck and back and can also help manage chronic lower back pain. A more sedentary lifestyle can increase the risk of developing chronic pain resulting in muscle atrophy, and fatigue as well as joint stiffness and immobility, all of which can be treated through exercise therapy. ${ }^{24}$

\section{Study rationale}

In modern society, there is an ever-increasing prevalence of sedentary behaviour (coupled with additional screen time), which leads to reduced physical activity and an increase in poor musculoskeletal health, especially during the COVID-19 pandemic. This paints a concerning picture for the future world we live in where more people may become sedentary, and subsequently, unhealthy. ${ }^{21}$

Previous studies regarding video gameplay, and its contribution to sedentary behaviour have solely focused on adolescents and the youth, but have neglected to include adults, who make up the greater proportion of video gamers. This limited research provides a challenge in us understanding whether or not seated video gameplay does actually contribute to sedentary behaviour. On the other hand, musculoskeletal health and more specifically poor musculoskeletal health, have been associated with sedentary behaviour. Most studies have focused on musculoskeletal health within the workplace. There currently exists limited data on recreational sedentary hobbies such as video gaming which could potentially pose a risk in one developing poor musculoskeletal health.

Therefore, the primary aim of this research was to determine the physical activity levels of non-active video gamers, and to determine how much other time was spent with sedentary behaviours, both in recreational and occupational domains. The secondary aim of this study was to establish whether or not seated video gameplay, a sedentary hobby, contributed to increased prevalence of physical inactivity, and poor musculoskeletal health among adult South African video gamers. In order to do this, it was necessary to investigate the lifestyles, gaming habits and musculoskeletal health of this population.

\section{METHODS}

\section{Study design}

This is a descriptive research study which employed an analytical research study design.

\section{Study participants}

Since most video gamers $(70 \%)$ are over the age of $18,{ }^{2}$ it was a requirement that participants were over the age of 18 years in order to participate in this research study. More than 100 participants were deemed adequate for this population size.

\section{Inclusion criteria}

Willing participants qualified for the study if they met the following requirements:

- South African.

- Over 18 years of age.

- Access to $\mathrm{Wi}-\mathrm{Fi} /$ internet.

- Video gaming performed 5 hours or more per week. ${ }^{25}$

\section{Exclusion criteria}

Willing participants did not qualify for the study if they met the following characteristics:

- Younger than 18 years of age.

- Video gaming performed less than 5 hours/week. ${ }^{25}$

- Not currently living in South Africa.

- No access to Wi-Fi/internet.

\section{Instruments}

The instrument used for this study was the I-PAQ. The I-PAQ has been approved and adopted in 12 different countries, both in developed and low-income and middle-income countries, and is found to provide accurate and valid data both in rural and urban areas. Access to the survey was online, with Google being used via a link that was embedded as a GoogleForms questionnaire which was sent to the participants during the COVID-19 pandemic. 


\section{Data collection}

Participants had access to the questionnaire via an internet link. Participants could use any electronic device that had access to the internet. The link directed participants to a personalised GoogleForm page, which was used to gather the data for the research study. The first page provided participants with information regarding the research such as the purpose of the study and what was expected of the participant (information letter). It also required participants to confirm their involvement in the study, thereby giving providing their consent online. Once this was completed, participants could participate in the research questionnaire which took approximately 20-25 min to complete. The information collected from the online questionnaires was subsequently stored in the GoogleForms backend cloud storage, via GoogleSheets. Google Sheets was exported as a Microsoft Excel Sheet (xslv.) and was set up to generate the statistics from the study.

\section{Data analysis}

Both descriptive (using means and SD) and inferential (independent t-tests) statistics were employed as part of the analysis, using SPSS (IBM, V.26) software. The level of significance was set at $\mathrm{p}<0.05$.

\section{Ethical considerations}

Ethical approval for the study was granted by the university's Faculty of Health Sciences Research Ethics Committee and informed consent was obtained by all participants prior to participating in the study. This research study also conformed to the World Medical Association Declaration of Helsinki on Ethical Principles for Research Involving Human Subjects. ${ }^{26}$

\section{RESULTS}

\section{Demographical characteristics}

There were 102 participants who took part in this study. The questionnaire was divided into three sections. Part 1 gathered an understanding of participants' gaming experience focusing on time spent playing, the devices most frequently used, breaks taken and sedentary nature of gamification. Part 2 focused on understanding the participants' lifestyle and identifying overall physical activity levels in relation to sedentary behaviour. Finally, part 3 obtained an understanding of participants' musculoskeletal health parameters in conjunction with their posture habits, musculoskeletal pain experienced while gaming, as well as how participants dealt with the pain.

\section{Understanding gaming behaviour}

Of the 102 participants, 42 stated $(41.2 \%)$ that they played for 5 hours or more on an average weekday $(n=42$, $41.2 \%$ ).

Most gamers seem to take breaks between gameplay, with the general trend being approximately 1 hour per break. This was an open-ended question which was

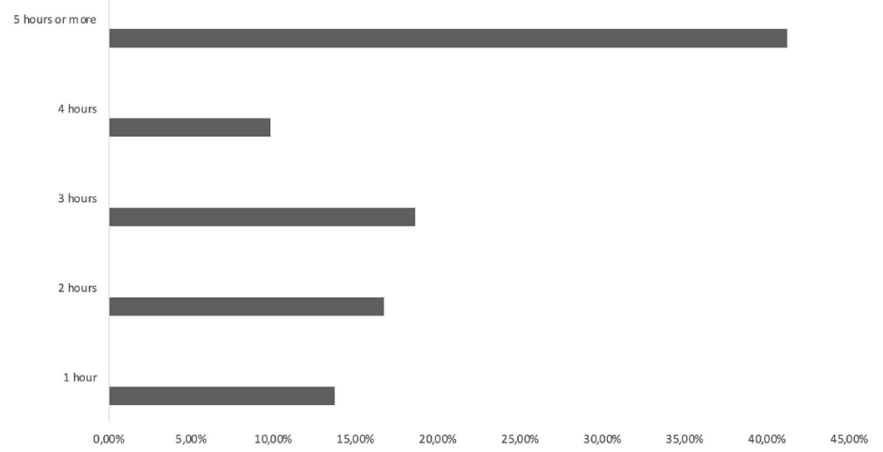

Figure 1 Gamers response to the following question: On average per day, how many hours do you spend on seated video games in the week?

further accompanied by the reasoning behind the breaks, which was mostly to get food or sleep.

In terms of days played during the week, $41.2 \%$ of participants stated that they played every day of the week $(\mathrm{n}=42,41.2 \%)$ with a further $18.6 \%$ of participants playing at least 4 days/week ( $\mathrm{n}=19,18.6 \%)$ (figure 1). These findings demonstrate a high frequency for weekday gaming, combined with the average number of hours played on an average weekday, which shows a considerable amount of time spent playing games during the week, and at high-frequency levels among the participants.

Most participants video game seated or reclined $(n=98$, $96.1 \%, \mathrm{p}=0.01$ ), with only four participants stating that they engage in some form of active video gaming. The results show the popularity for more traditional seated video gaming instead of active video gaming. Participants were split between using a console device (Xbox, Playstation, etc), or personal computer/laptop with $47.1 \%$ of participants selecting consoles as their most frequently used gaming device $(\mathrm{n}=48,47.1 \%)$ and $39.2 \%$ of participants selecting the $\mathrm{PC} /$ laptop as their most frequently used gaming device $(n=40,39.2 \%)$ (figure 2$)$.

\section{Understanding participants' lifestyle}

Regarding physical activity levels, $87.3 \%$ of participants stated that they participate in moderate-to-vigorous intensity physical activity such as brisk walking $(n=89,87.3 \%$,

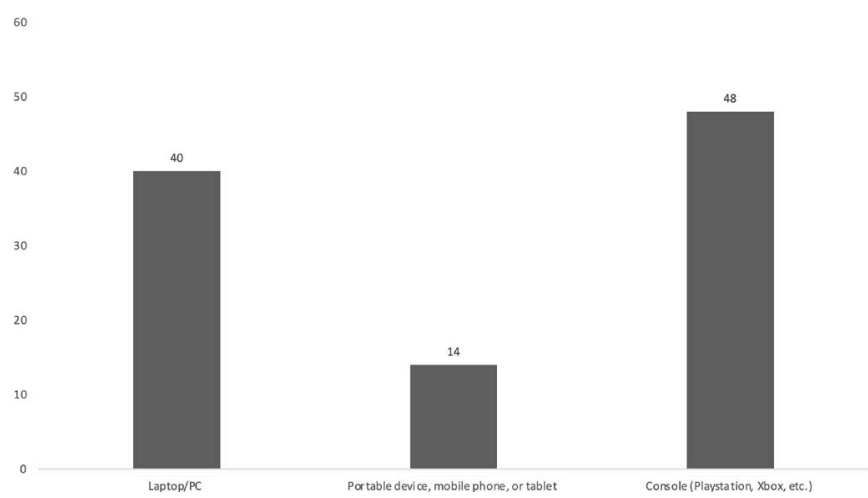

Figure 2 Number of participants and type of device most frequently used. 


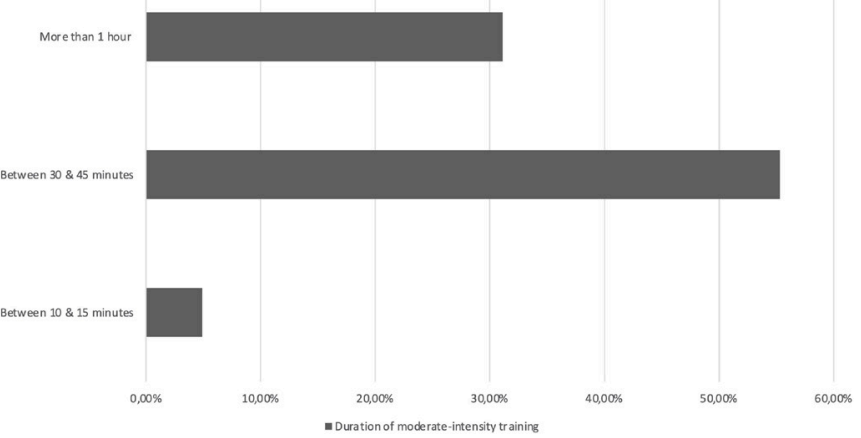

Figure 3 Duration of moderate-intensity physical activity.

$\mathrm{p}=0.02$ ), while $12.7 \%$ stated that they do not engage in any moderate-intensity activity.

For time spent on moderate-intensity activity, the most common choice was between 30 and $45 \mathrm{~min}$ (among $61.3 \%$ of participants) ( $\mathrm{n}=57,61.3 \%)$. A further $33.3 \%$ of participants stated that they engage in more than an hour of this form of activity on a typical day $(n=31$, $33.3 \%$ ) (figure 3 ).

For physical activities that are considered vigorous such as running or playing soccer, $72.5 \%$ stated that they engaged in this form of activity $(\mathrm{n}=74,72.5 \%)$, with the remaining $27.5 \%$ of participants stating that they did not $(\mathrm{n}=28,27.5 \%)$.

The majority of participants $(\mathrm{n}=64,62.7 \%)$ stated that they spent most of the working week seated or reclined, with a further $20.6 \%$ stating that they spend half the working week seated or reclined $(n=21,20.6 \%)$. The rest of the participants stated that they spend less than half the week seated or reclined $(\mathrm{n}=17,16.7 \%)$ (figure 4).

Furthermore, participants ingest a combination of snacks and beverages while video gaming $(n=37,53.6 \%)$. This was closely followed by the beverages only option (40.6\% of participants). Only $5.8 \%$ of participants selecting snacks only. It seemed that energy drinks and coffee were a common selection, followed by alcoholic beverages such as beer chosen among the beverages consumed while gaming. In terms of food, it seemed most participants consumed chips or crisps, or sugary foods such as chocolate or sweets.

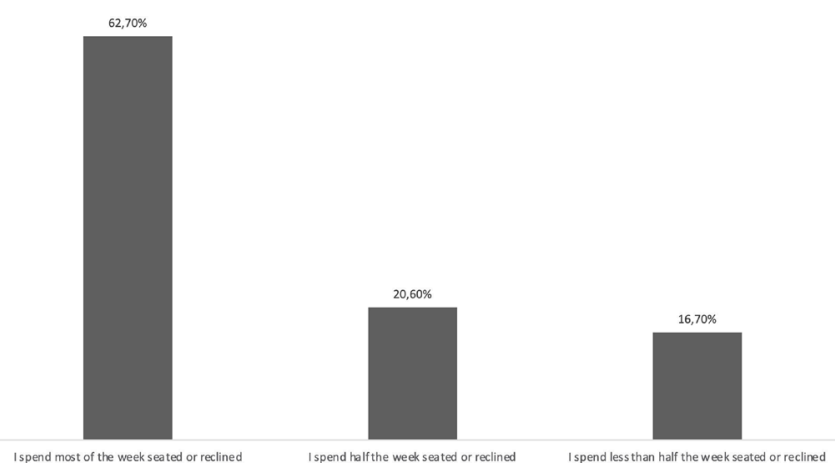

Figure 4 Sedentary behaviour of participants during the week.

\section{Understanding participants' musculoskeletal health}

Prevalence of pain while gaming

In terms of pain experienced while gaming, it was evenly split with just over half the participants stating they experienced some form of pain while gaming $(n=55,53.9 \%)$, with the other 47 participants stating that they experienced no pain while gaming. Of the participants who experienced pain, $67.2 \%$ claimed the pain was present in their back, while $40.6 \%$ stated they experienced the pain in the neck. Movement such as walking around or standing was common for pain relief, followed by stretching.

\section{Mechanism of pain}

It was evident that most participants who stated that they experienced pain, described it as an aching pain $(n=44$, $83 \%$ ), followed by burning pain (7.5\% of participants). The majority of the participants who stated they experienced pain, stated it was not accompanied by pins and needles $(n=71,84.5 \%)$ with only $15.5 \%$ of those participants stating that their pain was accompanied by pins and needles.

\section{Aetiology of pain}

When asked about whether or not participants experienced headaches or dizziness, $24.5 \%$ stated that they did $(\mathrm{n}=24,24.5 \%)$, while $75.5 \%$ stated that they did not. This indicates that most of the gamers do not experience any headaches or dizziness associated with gameplay $(n=74$, $75.5 \%)$.

\section{Posture}

It was evident that being conscious of good posture was only prioritised by just under half of the participants with only $46 \%$ stating that they are aware of good posture $(n=46,46 \%)$, while $54 \%$ stated that they were not aware of good posture during gaming $(n=54,54 \%)$. A vast majority stated that they did not make use of anything to help aid in good posture.

\section{DISCUSSION}

The past-time recreational activity of video gaming in the seated form is a rapidly expanding sedentary hobby, thought to result in a decrease in physical activity, and an increase in a more sedentary lifestyle having negative health implications. Studies have shown increased sedentary behaviour to be associated with the development of certain cancers, overweight and obesity, diabetes, poor posture and poor musculoskeletal health. This study attempted to corroborate the negative implications of prolonged seated video gaming of earlier studies by analysing adult South African video gamers and their gaming habits, lifestyle habits and musculoskeletal health.

When comparing time spent video gaming with physical activity levels of participants, the major findings were that most gamers tend to game for a considerable amount of time on a given day, and the majority tend to play most days of the week. A 2019 study found the 
average amount of hours spent gaming is 7.11 hours a week among 4500 adults from nine different countries (France, Germany, India, Italy, Japan, Singapore, South Korea, the UK and the USA). ${ }^{5}$ It was further found that the mobile phone was the most popular to game on, followed by the personal computer and subsequently the console (Xbox and Playstation). This contradicts the data collected in this study where the findings were that console or television-based gaming was the most popular device followed by the personal computer and then the mobile phone gaming. This study also found that almost all of the participants engage in sedentary/seated video gamification.

\section{Comparison between gaming behaviour and physical activity levels}

Findings from this study demonstrate that video gamers in South Africa are physically active and maintain adequate amounts of physical activity bouts, despite the high frequency and duration of gaming. This is not supported by a previous study on sedentary gaming among adolescent men in Finland. ${ }^{27}$ It was discovered that individuals who played more than 3 hours a day had poorer health habits or behaviours than those who played less relating to physical fitness levels, body mass and diet. This may be due to the varied demographics and could potentially show that South Africans are more physically active than other countries.

This study finds no correlation between prolonged seated video gaming and physical inactivity. Most of the participants were physically active and engaged in either moderate-intensity (cycling, brisk walking, swimming, volleyball, etc) or vigorous-intensity physical activity (running, football, etc) or both, despite their prolonged gamification. This is validated in a study that focused on procrastination and video gaming, which demonstrated no correlation between increased video gaming and procrastination. ${ }^{28}$ Furthermore, findings suggest that the duration of the moderate and vigorous physical activities on a given day is adequate for the majority of the participants. The findings are supported by the ACSM recommendations for exercise testing which concurs that getting $150 \mathrm{~min}$ of moderate intensity exercise or $75 \mathrm{~min}$ of vigorous-intensity exercise greatly diminishes the risk of developing certain NCDs and metabolic diseases. ${ }^{16}$

\section{Comparison between sedentary behaviour and physical activity levels}

Another study has discovered a direct link between long sedentary working hours with increased physical inactivity. However, findings from this study show that this may not be the case among participants. ${ }^{29}$ The majority spend half or more than half of the week seated/reclined. Despite this, physical activity levels reported in this study show weekly frequency of both moderate and vigorous physical activity.

\section{Musculoskeletal health among participants}

Sedentary behaviour has long been associated with musculoskeletal pain, with most studies focusing, particularly on adolescents. Findings from this study show a significant portion of participants stating that they experienced pain when they video game, particularly in the neck and back. These findings are reinforced by another study which found a direct association with musculoskeletal pain and prolonged video gaming among adolescents between the ages of 13 and $18 .^{30}$ The study further finds that reports of musculoskeletal pain during adolescence could continue into adulthood unless sedentary habits, in this case, video gaming changes.

The nature of the musculoskeletal pain was vastly described by participants as being an aching pain and the two most common sites were in the back and neck. This could be attributed to the fact that prolonged sitting, results in stiffness of the back and neck muscles, therefore resulting in an aching pain after prolonged video gaming. ${ }^{31}$ An ergonomic chair may be effective in the lower back and trunk support to aid in adequate posture while gaming. An evaluation of an ergonomic chair has shown promise with the trunk and forearm being supported which is desirable in reducing musculoskeletal pain after prolonged sitting time. ${ }^{32}$

\section{Posture}

Findings from this study suggest that most participants disregard posture when video gaming. This may be due to the fact that video gaming can require a substantial amount of concentration where posture (or maintaining good posture) is not prioritised. Some participants stated that they adjusted their seating and height to maintain an upright position while seated. However, this was only a select few.

I lock the seat in the upright position. I make my screen face more upwards to force me to sit upright to see what's going on and finally, I allow for light to hit the screen to create glare so that I am forced to sit upright to block the light to see what's going on.

One of the participant's responses to a question regarding posture and how they maintain good posture. This response represented the minority of participants.

\section{Strengths and limitations}

The strengths of this research study are owed to the specificity of South African video gamers. This, however, is not generalisable when compared with other low-to-middle income countries relating to gaming behaviour, lifestyles and musculoskeletal health. These data could further contribute to a greater understanding of health profiles and the effect it has among gaming individuals. Another strength was that the questionnaire was based on a validated questionnaire, the I-PAQ. This questionnaire has been widely used and adopted to study physical activity levels among individuals from different populations, globally. A limitation to this research study is that it was 
limited to South African video gamers, where conclusions can only be drawn from this group, and not regarding associations of physical inactivity and sedentary behaviour on a broader spectrum. In addition, the sample size was not entirely adequate, and a greater number of individuals would have added more insight to the current study. Another limitation to this research study was the subjective nature of the quantitative data collected, as opposed to objective testing such as body mass and physical fitness testing. Such objective measures were planned but could not be performed due to the lockdown levels imposed at the time during the COVID-19 pandemic.

\section{CONCLUSION}

Findings from this study show that high frequency and duration for video gaming among gamers does not affect physical activity for moderate and vigorous intensities, but does affect musculoskeletal pain. Furthermore, the study finds the console to be the most popular device to game on, followed by the personal computer and the phone. Findings also suggest that individuals are not conscious of good posture, and the majority of participants also experience pain while gaming. Further research is required to gather further insight among video gamers and its effects on one's physical and mental health. Determining whether or not video gaming has a significant effect on lifestyle, sedentary habits and musculoskeletal health, is also important, especially in the context of the COVID-19 pandemic where sedentary behaviour has likely increased. Further research should also be expanded with the aim of assisting policymakers to create guidelines to increase physical activity and reduce sedentary behaviour in population groups for various sectors and industries, and not just for video gamers.

\section{Twitter Habib Noorbhai @Habib_Noorbhai}

Contributors NH performed the data collection and analysis of the participants and wrote the manuscript. HN conceptualised the study, assisted in the design of the study and data analysis, wrote the manuscript and provided oversight on the project.

Funding The authors have not declared a specific grant for this research from any funding agency in the public, commercial or not-for-profit sectors.

\section{Competing interests None declared.}

Patient and public involvement Patients and/or the public were not involved in the design, or conduct, or reporting, or dissemination plans of this research.

\section{Patient consent for publication Not required.}

Ethics approval Ethical approval for the study was granted by the University of Johannesburg, Faculty of Health Sciences Research Ethics Committee (REC-4412020). Informed consent was obtained by all the participants prior to participating in the study. This research study also confirmed to the World Medical Association Declaration of Helsinki on Ethical Principles for Research Involving Human Subjects.

Provenance and peer review Not commissioned; externally peer reviewed. Data availability statement Data are available upon reasonable request. N/A. Open access This is an open access article distributed in accordance with the Creative Commons Attribution Non Commercial (CC BY-NC 4.0) license, which permits others to distribute, remix, adapt, build upon this work non-commercially, and license their derivative works on different terms, provided the original work is properly cited, appropriate credit is given, any changes made indicated, and the use is non-commercial. See: http://creativecommons.org/licenses/by-nc/4.0/.
ORCID iD

Habib Noorbhai http://orcid.org/0000-0001-9464-6854

\section{REFERENCES}

1 D'Argenio AM. Statistically video games are now the most popular and profitable form of entertainment, 2018. Available: https://www. gamecrate.com/statistically-video-games-are-now-most-popularand-profitable-form-entertainment/20087 [Accessed 27 Feb 2021].

2 Nielsen. Video game console ownership in US, 2018. Available: https://www.marketingcharts.com/cross-media-and-traditional/ videogames-traditional-and-cross-channel-82362/attachment/ nielsen-video-game-console-ownership-feb2018 [Accessed 27 Feb 2021].

3 WePC. WePC, 2019. Available: https://www.wepc.com/news/videogame-statistics/\#video-gaming-industry-overview [Accessed 27 Feb 2021].

4 Kemp C, Pienaar P, Rae D. Brace yourselves: esports is coming. S Afr $J$ sports med 2020;32:1-2.

5 Limelight Networks. Market research the state of online gaming, 2019. Available: https://img03.en25.com/Web/LLNW/\% 7B02ca9602-173c-43a4-9ee1-b8980c1ea459\%7D_SOOG2019_ MR_8.5x11.pdf [Accessed 7 Feb 2021].

6 Simons M, Chinapaw MJM, Brug J, et al. Associations between active video gaming and other energy-balance related behaviours in adolescents: a 24-hour recall diary study. Int J Behav Nutr Phys Act 2015;12:32.

7 Sedentary Behaviour Research Network. SBRN, 2019. Available: https://www.sedentarybehaviour.org/what-is-sedentary-behaviour/ [Accessed 28 Feb 2021].

8 Edwardson CL, Gorely T, Davies MJ, et al. Association of sedentary behaviour with metabolic syndrome: a meta-analysis. PLoS One 2012;7:1.

9 American Heart Association. The American heart association, 2019. Available: http://www.heart.org/HEARTORG/Conditions/ More/MetabolicSyndrome/About-Metabolic-\%20Syndrome_UCM 301920_Article.jsp\#.XZCDWSgzaDI [Accessed 29 Feb 2021].

10 Tomioka K, Iwamoto J, Saeki K, et al. Reliability and validity of the International physical activity questionnaire (IPAQ) in elderly adults: the Fujiwara-kyo study. J Epidemiol 2011;21:459-65.

11 Hopkins M, Horner K, King N. Exercise, appetite control, and body weight regulation. in: physical activity, exercise, sedentary behavior and health. Tokyo: Springer, 2015: 123-6.

12 Noorbhai MH. A public health approach to increase physical activity and health education: the Biokinetic humanitarian project. Afr J Phys Health Ed Recr Dance 2013;19:993-8.

13 Rimmer JH, Rowland JL, Yamaki K. Obesity and secondary conditions in adolescents with disabilities: addressing the needs of an underserved population. J Adolesc Health 2007;41:224-9.

14 World Health Organization. Diet and physical activity: a public health priority. [Online]; 2019. Available. Available: https://www.who.int/ dietphysicalactivity/pa/en/ [Accessed 29 February 2021].

15 Sardo Molmenti CL, Hibler EA, Ashbeck EL, et al. Sedentary behavior is associated with colorectal adenoma recurrence in men. Cancer Causes Control 2014;25:1387-95.

16 American College of Sports Medicine. Benefits and risks associated with physical activity. In: ACSM's Guidelines for Exercise Testing and Prescription. 9th ed. Philadelphia: Lippincott Williams \& Wilkins, 2014.

17 Cleland CL, Hunter RF, Tully MA, et al. Identifying solutions to increase participation in physical activity interventions within a socio-economically disadvantaged community: a qualitative study. Int J Behav Nutr Phys Act 2014;11:1-9.

18 Ryan DJ, Wullems JA, Stebbings GK, et al. Reliability and validity of the International physical activity questionnaire compared to calibrated accelerometer cut-off points in the quantification of sedentary behaviour and physical activity in older adults. PLoS One 2018;13:e0195712-7.

19 Hallgren M, Owen N, Stubbs B. Cross-Sectional and prospective relationships of passive and mentally active sedentary behaviours and physical activity with depression. Br J Psych 2020;214:1-7.

20 Lederman O, Grainger K, Stanton R, et al. Consensus statement on the role of accredited exercise Physiologists within the treatment of mental disorders: a guide for mental health professionals. Aust Psych 2016;24:347-51.

21 Mahdavi SB, Kelishadi R. Impact of sedentary behavior on bodily pain while staying at home in COVID-19 pandemic and potential preventive strategies. Asian J Sports Med2020;11:e103511.

22 Bennie JA, Pedisic Z, van Uffelen JGZ, et al. The descriptive epidemiology of total physical activity, muscle-strengthening exercises and sedentary behaviour among Australian adults - results 
from the National nutrition and physical activity survey. BMC Public Health 2015;16:73.

23 Gaur L. How to avoid lower back pain in a sitting job, 2019. Available: https://www.indianbureaucracy.com/how-to-avoid-lowerback-pain-in-a-sitting-job/ [Accessed 16 Mar 2021].

24 Yang YJ. An overview of current physical activity recommendations in primary care. Korean J Fam Med 2019;40:135-42.

25 Berard AV, Cain MS, Watanabe T, et al. Frequent video game players resist perceptual interference. PLoS One 2015;10:3.

26 World Medical Association. World Medical association Declaration of Helsinki: ethical principles for medical research involving human subjects. JAMA 2013;310:2191-4.

27 Puolitaival T, Sieppi M, Pyky R, et al. Health behaviours associated with video gaming in adolescent men: a cross-sectional populationbased MOPO study. BMC Public Health 2020;20:1-8.
28 Nordby K, Løkken RA, Pfuhl G. Playing a video game is more than mere procrastination. BMC Psychol 2019;7:33.

29 Mansoubi M, Pearson N, Biddle SJH, et al. The relationship between sedentary behaviour and physical activity in adults: a systematic review. Prev Med 2014;69:28-35.

30 Hellström C, Nilsson KW, Leppert J, et al. Effects of adolescent online gaming time and motives on depressive, musculoskeletal, and psychosomatic symptoms. Ups J Med Sci 2015;120:263-75.

31 Boukabache A, Preece SJ, Brookes N. Prolonged sitting and physical inactivity are associated with limited hip extension: a crosssectional study. Musculo Sci Prac 2021;51:102282.

32 Lim SY, Won BH. Ergonomic evaluation of Trunk-Forearm support type chair. J Erg Soc of Korea 2014;33:143-53. 